\title{
LAS NUEVAS NECESIDADES FORMATIVAS EN EL MARCO DE LA DIGITALIZACIÓN Y DEMÁS AVATARES 4.0 (LA AGENDA 2030 Y LOS OBJETIVOS DE DESARROLLO SOSTENIBLE COMO HERRAMIENTAS DOCENTES)
}

The New Training Needs in the Framework of Digitalization and Other Avatars 4.0 (Agenda 2030 and Sustainable Development Goals as Teaching Tools)

M. a Gema Quintero Lima

Universidad Carlos III de Madrid

\section{ABSTRACT}

En apariencia, los primeros análisis de la digitalización y la robotización de la economía parecen coincidir en que tendrá efectos negativos para el empleo: en la medida en que ciertas actividades puedan ser sustituidas totalmente por procesos digitales o por sujetos robotizados, se hace prescindible ya la presencia total o parcial de trabajadores en las empresas. Solo indirectamente se hace referencia a la incidencia de esos nuevos procesos en el estatuto jurídico del trabajador, en las relaciones laborales colectivas y en la revisión de los sistemas de protección social.

Desde septiembre 2015 se han activado desde las Naciones Unidas los Objetivos de Desarrollo Sostenible (ODS), que pueden ser las nuevas directrices de inspiración para nuevas herramientas de gestionar el cambio, o para nuevos contenidos de herra- 
mientas clásicas. La legislación y la negociación colectiva son destinatarias de algunos mandatos (referidos a regulaciones de salarios y demás condiciones decentes, son los ejemplos más inmediatos), de ahi que sea preciso insertar nuevas vias de reflexión en la docencia del Derecho Social 4.0. Al mismo tiempo, los ODS son susceptibles de actuar como acelerantes del aprendizaje, por lo que de estimulantes tienen en el ámbito material del Trabajo Decente y del Desarrollo Sostenible.

Palabras clave: Innovación Docente, Cuarta Revolución Industrial; Agenda 2030, trabajo decente, necesidades formativas.

The first analysis of digitalisation and the robotization of the economy seem to converge in the idea that Revolution 4.0 will have negative effects on employment: if certain activities can be completely replaced by digital processes or by robotic subjects, then it is possible that workers in companies are totally o partially replaced. There are just only indirect references to the impact of these new processes on the legal status of the worker, on collective labor relations and on the revision of social protection systems.

Since September 2016 the Sustainable Development Goals (SDG) have been activated from the United Nations, which may be new inspiration guidelines for new tools to manage change, or for new contents of classic tools. Legislation and collective bargaining are the recipients of some mandates (referring to salary regulations and other decent conditions, are the most immediate examples) hence it is necessary to insert new ways of rethinking the teaching of Social Law 4.0. At the same time, the $S D G$ are likely to act as accelerators at learning processes, so that they are stimulating frameworks in the material scope of Decent Work and Sustainable Development

Keywords: Teaching Innovation, Fourth Industrial Revolution; 2030 Agenda, decent work, training needs. 


\section{SUMARIO}

Sumario. 1. Los cambios en el Paradigma del Trabajo como punto de partida. 1.1. La Revolución Tecnológica 4.0. 1.2. Los interrogantes sobre la validez de los conceptos clásicos del Derecho Social. 2. El marco de reflexión internacional sobre el Futuro del Trabajo: el Trabajo Decente y Sostenible. 2.1. Un prius: el escenario de la Europa Digital. 2.2. El debate sobre el futuro del Trabajo a iniciativa del Director de la Organización Internacional del Trabajo. 2.3. La Agenda ONU 2030 y los Objetivos de Desarrollo Sostenible como Axiomas duraderos de gestión necesaria del cambio. 3. El papel de las Universidades como catalizadores de la investigación y de la formación en Objetivos de Desarrollo Sostenible en y para el Trabajo Decente. 4. Los estudios de Relaciones Laborales y los ODS. 4.1. Los estudiantes como Agentes. 4.2. Los ODS como marcos éticos del aprendizaje de los estudiantes. 4.3. Los ODS como herramientas de Innovación Docente. 4.3.1. Nuevos contenidos: la afectación de los programas. 4.3.2. Fundamentos profesionales éticos. 4.3.3. La motivación de los estudiantes. 5. Bibliografía.

\section{Los cambios en el Paradigma del Trabajo como punto de partida}

\subsection{La Revolución Tecnológica 4.0}

Desde una percepción cíclica de la organización del trabajo desde la perspectiva socio-jurídico-económica, se inserta la idea de que se avizora (o ya llegó) la Cuarta Revolución Industrial. Que parece que, superada la revolución geográfica de la Globalización, ahora se conforma como una nueva era de Robotización y Digitalización de la Economía y, por ende, del Trabajo.

Sin que se hayan resuelto aún completamente las cuestiones jurídicas ligadas a la evaporación de las territorialidades del Derecho y la necesaria efectividad de las normas mínimas del trabajo en un mundo global con agentes multinacionales y estados de dispar protección jurídico-laboral (Lantarón, 2017), se ha venido llamando la atención en los últimos meses sobre la posibilidad de que el trabajo deje de ser eminentemente humano, de que el trabajo humano quede obsoleto y de que el desempleo se convierta en la regla general de las sociedades modernas (Ford, 2016).

En el debate amplio, desde disciplinas muy dispares, se discute, en síntesis, sobre si en la actualidad se produce la destrucción, la creación o la mutación del 
empleo (López, 2016), si se produce una destrucción creativa o si, por el contrario, se está generando una situación inusitada, dado que los avances tecnológicos se aplican ahora a todo el espectro profesional, en todos los niveles formativos, sin que los procesos de reconversión industrial vayan a ser ahora posibles.

En efecto, y sin que sea posible profundizar demasiado en ese debate, desde sectores experimentales (ingeniería robótica, computación) y económicos de cierto signo, se avanza una visión determinada del futuro del trabajo, que pivota sobre el potencial destructor de empleo de los robots y de la Inteligencia Artificial (IA).

En efecto, varios estudios enjundiosos desde las perspectivas económica y de la administración de empresas, con o sin lobbies presentes, proclaman el fin del trabajo humano y su sustitución por la Inteligencia Artificial (IA), el trabajo robotizado, en distintas manifestaciones (cadenas de robotización sofisticada, presencia de Bots humanoides, procesos digitales de producción de servicios, sistemas de fabricación a partir de impresión 3D...) (McKinsey Institute, 2017; WEF, 2017; Frey, 2017).

En ese esquema, fluye una idea catastrófica, la de que se ha de producir un cambio en el modelo económico clásico.

De una parte, habrá un mundo hiperautomatizado, en el que se producirán excedentes ingentes de mano de obra que conducen a un escenario de crisis social de dimensiones aún desconocida a partir de niveles de desigualdad extremos (propietarios, inversores y empleados de alto nivel versus desempleados descualificados que se encuentran por debajo del umbral de pobreza).

De otra parte, se provocaría una fuerte deflación económica porque, si bien la robotización genera un alto nivel de productividad, no hay consumidores suficientes (máquinas/robots/algoritmos no son consumidores) y los trabajadores no tienen capacidad adquisitiva. En síntesis, se produciría la quiebra del modelo de economía de mercado, que solo sería superable a partir de nuevas formas de protección social ancladas sobre algún género de renta mínima universal (Ford, 2016).

Esa posición, no obstante, en ese mismo sector de pensamiento en el campo de la ingeniería y de las organizaciones, encuentra también freno. Porque se estima que la incidencia de la robotización y del desarrollo de la IA no es cuantitativamente clara aún, y tampoco pueden apuntarse certeramente sus ámbitos temporales (Freeman, 2015). En este sentido, si bien es cierto que los avances tecnológicos son imparables y se acumulan exponencialmente en ciertos sectores, también es cierto que afecta de modo desigual a los distintos Estados, dentro de cada país a los distintos sectores productivos, a los distintos tipos de empresas y a las distintas regiones geográficas, y en todo caso es intrínseca a los distintos procesos productivos. De suerte que los datos sobre la afectación del empleo 
agregado, sobre la sustitución de trabajadores, sobre el engrose de las bolsas de desempleados tecnológicos han de ser tomados con mucha cautela jurídica. Porque se entiende que hay un proceso de cambio amortiguado que será especialmente pausado en Espańa, en la medida en que presenta, desde un punto de vista estructural, ciertas resistencias a la Digitalización/robotización acelerada (Berger, 2016; Ministerio de Industria, 2015).

Por esta razón, merecen mayor atención otras líneas de pensamiento. Porque, en paralelo a las anteriores, sin tanta parafernalia de ciencia ficción, hay ciertos trabajos doctrinales (Mercader, 2017) que aventuran la afectación de la realidad del trabajo y la necesaria revisión de su regulación, como consecuencia de la plausible sustitución total/parcial, inmediata/progresiva, de procesos manuales por procesos digitalizados o robotizados, en los que ha de convivir el trabajo humano con las distintas formas de Inteligencia Artificial, como compañeras o como instrumentos (herramientas o mecanismos de sustitución de ciertas tareas); como coadyuvantes en suma (Kaplan, 2016).

En esta segunda visión menos sensacionalista, tiene sentido adelantar la necesidad de cambios conceptuales referidos al Trabajo y a su regulación jurídica como ha venido siendo construida desde la Revolución Industrial clásica. Y se ha de admitir como hipótesis de partida que en las reflexiones desde el Derecho Social no cabría dejarse llevar por la falacia de los tres determinismos del Futuro del Trabajo (Barreto, 2017) ${ }^{1}$.

\subsection{Los interrogantes sobre la validez de los conceptos clásicos del Derecho Social}

A partir de esas cautelas, sí parece poco discutible, a partir de la observancia empírica, que, en primer lugar, los conceptos nucleares de trabajador y empresario en la relación laboral se desdibujan cuando las plataformas digitales permiten una oferta de bienes y servicios directa en la que los agentes presentes son el cliente y el oferente. En este sentido, pareciera que el esquema jurídico-laboral clásico se desdibuja y se reconduce a formas tradicionales de Derecho Privado de arrendamientos de obra o servicio.

El fenómeno Uber, pero también todas aquellas nuevas iniciativas de puesta a disposición de los usuarios finales, a través de aplicaciones digitales (Apps di-

\footnotetext{
${ }^{1}$ El primer determinismo consiste en considerar a la tecnología como un factor novísimo de cambio radical, una «disrupción»; el segundo se traduce en la idea de que la implantación de las nuevas tecnologías demanda que deba prescindirse de toda regulación para que no sea enervado su proceso explosivo; y el tercero, tiene que ver con la opción metodológica misma, que entiende que el futuro del trabajo solo puede revisarse desde el punto de vista del efecto tecnológico en el Trabajo.

Algún autor (Barreto,2017) considera incluso que la exigencia de incidir en el aspecto tecnológico no es más que una estrategia conspirativa, más o menos hábil, para «obtener márgenes más amplios de libertad de mercado, todo lo cual no tendría nada de novedoso e innovadon.
} 
versas), de prestaciones más o menos profesionalizadas, han abierto un debate principal acerca de cuál haya de ser la forma jurídica que asuman esos intercambios, y de cuáles hayan de ser los derechos y obligaciones de los prestadores de servicios y de las eventuales plataformas digitales de intermediación. Los conceptos de ajeneidad y subordinación se invisibilizan, pero la demanda de derechos sociales se convierte en un objetivo finalista para categorizar esas nuevas relaciones jurídicas. $\mathrm{O}$ lo que es lo mismo; cuando la consecuencia de remitir a la autonomía de la voluntad la conformación jurídica de los derechos (referidos a tiempos de trabajo, remuneración o a las responsabilidades en caso de accidente o inactividad del prestador, por poner ejemplos) es la nihilización de la protección social individual, entonces se produce un esfuerzo conceptual por la reconducción al trabajador asalariado clásico, o al trabajador autónomo clásico, o al semi-nuevo Trabajador Autónomo Dependiente Económicamente (el TRADE de la Ley 20/2007, de 11 de julio del Estatuto del Trabajador Autónomo) (Todoli, 2017).

En segundo lugar, desde el punto de vista de relaciones laborales indubitadas, es decir, cuando se mantiene el esquema y contornos ex art. 1.1 ET, la introducción de las nuevas tecnologías dentro del objeto del contrato, o como contexto de la prestación de servicios, plantea nuevos interrogantes referidos al propio contenido de la relación laboral estatutaria y convencionalmente establecido; pero también referidos al control empresarial de la prestación laboral. Y con efectos ambivalentes, de mayor garantía jurídica de los derechos sociales o de debilitamiento potencial de los mismos.

En efecto, habría ciertos derechos laborales susceptibles de verse afectados: el tiempo de trabajo, y el salario y los sistemas de remuneración como efecto reflejo, son los elementos más obvios (1). Porque si hay nuevos procesos de producción favorecidos y optimizados a través de las nuevas tecnologías, las exigencias laborales ligadas a tiempo de trabajo pueden reducirse debido a distintas razones: porque la automatización de procesos posibilita tiempo de no-presencia de trabajadores, o porque cabe generalizar sistemas de teletrabajo estándares y nuevas formas de teletrabajo. Estas y otras manifestaciones, pueden favorecer procesos de conciliación de la vida laboral y personal/familiar, pero también puede provocar excedentes objetivos de trabajo disponible, o, en otro sentido, pueden ahondar en las cuestiones jurídico problemática ligadas a la (extra)territorialidad de la prestación de servicios transnacionales.

Por lo que respecta al salario, conectado con tiempo de trabajo, puede producirse la ruptura de ciertos ligámenes conceptuales entre el estatus de trabajador y el de sujeto protegido por las estructuras de Seguridad social. Desde el punto de vista de la redistribución, si cambian los sistemas de remuneración, los ingresos no son distribuidos uniformemente y siguen modelos de winner-takesall, esto tendría un reflejo importante en la financiación, primero, de los siste- 
mas públicos de protección, y condicionaría eventuales nuevos mecanismos de garantía de recursos alejados del principio de contributividad/ proporcionalidad, en aras de un nuevo protagonismo al principio de suficiencia.

Pero el propio objeto de la prestación (2) también toma especial presencia, en la medida en que: $1 .^{\circ}$ se hace precisa una nueva (o antigua) formación continua en el uso de nuevas tecnologías, y en la inserción en nuevos procesos productivos digitalizados o automatizados; del mismo modo la recualificación de trabajadores desempleados se erige como condiciones sine qua non de la adaptabilidad al cambio, 2. ${ }^{\circ}$ la productividad de cada trabajador tendría nuevos patrones de referencia, no humanos necesariamente; $3 .^{\circ}$ en consecuencia, las causas económicas, técnicas, organizativas y de producción se desdibujan y se vuelven aún menos corpóreas, con lo que ello entraña en términos de flexibilidad interna y externa en las relaciones laborales.

Por otra parte, los derechos fundamentales se exponen a nuevos riesgos (3), tanto los ligados a la seguridad y salud en el trabajo (donde hay nuevos riesgos, como los compañeros bots y la intensificación de la robotización, y las nuevas formas de riesgos psico-sociales ligados a la hiperconexión ${ }^{2}$, como los más propiamente inherentes a la personalidad (intimidad, honor, propia imagen, o libertad ideológica, de expresión), en la medida en los Big Data y ese Ojo del Gran Hermano permitirían un control biométrico complejo, por señalar un ejemplo ${ }^{3}$.

En ese sentido, paradójicamente, las nuevas tecnologías también tienen virtualidades positivas; porque esos Big Data y su gestión especializada, podrían servir para el control del cumplimiento de la normativa legal y convencional laboral. Y las herramientas digitales amplo sensu podrían ser herramientas inclusivas e integradoras de las diversidades clásicas, porque no tienen género y tienen el potencial de aminorar los efectos de distintas discapacidades físicas e intelectuales.

Además, las distintas aplicaciones en las que se sustentan las Redes Sociales se convierten en maximizadoras de la expansión de la autonomía colectiva, y de

2 Una reacción ya inmediata es el derecho a la desconexión (droit à la déconnexion) ya en vigor en Derecho francés desde el 1 de enero de 2017. Fue instaurado en el artículo L. 2242-8, 7. ${ }^{\circ}$ del Código de Trabajo, tras la reforma introducida por el art. 55 de la Ley n. ${ }^{\circ} 2016-1088$ de 8 de agosto de 2016 relativa al trabajo, a la modernización del dialogo social y al aseguramiento de los itinerarios profesionales.

3 Si bien las propias aplicaciones simples de los dispositivos electrónicos (ordenadores, móviles...) y el rastreo sencillo de los perfiles de los trabajadores en las redes sociales ya permiten supervisar sus comportamientos y ya han suscitado algunos problemas jurídicos en el ámbito del poder de dirección del empresario en el ámbito de la video-vigilancia (por ejemplo STS de 2 de febrero de 2017 (n. ${ }^{\circ}$ Recurso: 554/2016), o en el ámbito del despido fundado en el mal uso de los medios digitales que la empresa proporciona al trabajador y/o ligados a información obtenida de dispositivos informáticos en sentido amplio (por todas, STS de 6 de octubre de 2011 (n. ${ }^{\circ}$ Recurso: 4053/2010). 
la organización de formas participadas de transmisión de información (sustitutivos del tablón sindical clásico), de gestión de conflictos, o de prevención de los mismos.

Del mismo modo, la Administración Electrónica (de Seguridad Social, Servicios Públicos de Empleo, Agencia Tributaria, Sistemas Públicos de Salud, o Servicios Sociales) que comenzó en España su andadura hace ya una década, podría desenvolver nuevas funciones y maximizar las que ya realiza con mayor garantía para los usuarios (empresarios, trabajadores, representantes de ambos, gestores...) y mayores posibilidades de interacción con ciertos sujetos privados estratégicos (mutuas, fundaciones, colegios profesionales...).

Esta no es sino una panorámica vertiginosa de eventuales vicisitudes jurídicas de las nuevas realidades de la Industria 4.0 en clave de Empleo.

En este ámbito el Derecho Social (como concepto omnicomprensivo de las ramas clásicas reconocidas como Derecho Laboral, Derecho Sindical, Derecho de la Seguridad Social, Derecho Administrativo Laboral, Derecho de la Prevención de Riesgos laborales, Derecho Procesal Laboral etc.) habría de proceder a revisar conceptos clásicos, para revisitarlos y actualizarlos.

Pero, también, en su autopoiesis, puede incorporar una nueva composición utópica con nuevos esquemas de lege ferenda para la adaptación a los cambios que la Cuarta Revolución demanda. Y desde 2015 parece haber nuevas inspiraciones.

\section{El marco de reflexión internacional sobre el Futuro del Trabajo: el Trabajo Decente y Sostenible}

\subsection{Un prius: el escenario de la Europa Digital}

De un modo inespecífico, independientemente de las líneas reflexivas que se verán enseguida, se ha de llamar la atención sobre un hecho. Desde 2010, la Comisión Europea, en el Marco del Horizonte 2020, ha diseñado estratégicamente una Agenda Digital para Europa ${ }^{4}$. En el marco de un Mercado Único Digital se pretende universalizar la ciudadanía digital. Y en ese contexto general, el Mercado de trabajo, las Empresas y empleadores, los trabajadores y las Administraciones laborales y de Seguridad Social no quedan ajenos al proceso regionalizado de desarrollo tecnológico aplicado.

Siendo ésta una cuestión preliminar, sin embargo, interesan más el discurso internacional sobre el Futuro del Trabajo, que vas más allá de esa visión tecno-

\footnotetext{
${ }^{4}$ http://www.europarl.europa.eu/atyourservice/es/displayFtu.html?ftuId=FTU_5.9.3.html
} 
céntrica/ robóticocéntrica, y se escora hacia el Trabajo en sí mismo considerado, si bien en su contexto revolucionado 4.0.

\subsection{El debate sobre El futuro del Trabajo a iniciativa del Director de la Organización Internacional del Trabajo}

La necesidad de diseñar nuevas líneas de abordaje del Trabajo no es algo inherente a la digitalización ni al siglo XXI. Es mucho anterior. Puesto que la Globalización ya puso de manifiesto que los Estados no podían contener la vis expansiva de las empresas cuando éstas deciden fraccionar sus procesos productivos y descentralizarlos más allá de las fronteras iniciales de la actividad empresarial. Y tampoco alcanzaban a proteger los derechos de los trabajadores de la empresa de origen matriz, ni los de los trabajadores de destino. Ni los Estados de destino en ocasiones alcanzaban tampoco a hacerlo respecto de sus trabajadores asentados en su territorio nacional.

El Derecho del Trabajo nacional se ha encontrado, desde hacer bastante tiempo entonces, preso de sus fronteras. Si bien, regionalmente, en el caso de España y la Unión Europea, se había venido diseñando ciertas fórmulas de determinación de la ley aplicable al contrato y a eventuales procesos judiciales (los Reglamentos (CE) de Roma, y Bruselas, en sus versiones sucesivas, respectivamente, I y II, y I, II y IIbis), de idéntico modo las fronteras de la región han frenado la efectividad universal de estándares de protección relativamente altos, más allá del ámbito comunitario y de su área de influencia.

De ahí que se haya de prestar atención a otro núcleo normativo que circunscribe al anterior: el Derecho Internacional del Trabajo stricto sensu, el gestado y promocionado desde la Organización Internacional del Trabajo (OIT). Así, el Trabajo, si bien ha sido objeto recurrente de regulación en los distintos ordenamientos nacionales, también ha sido en paralelo, aunque paradójicamente con menor visibilidad, objeto del Derecho Internacional del Trabajo gestado por y emanado de, desde 1919, de la Organización Internacional del Trabajo (OIT). Desde entonces la idea de la Paz Mundial a partir de la Justicia Social ha regido la actividad normativa internacional relativa al trabajo en sus distintas vertientes.

No obstante su apariencia de ordenamiento supranacional potente, la mayor fortaleza del sistema económico-financiero mundializado, supone un límite, ya no territorial, sino estructural, referido a la coercitividad limitada de las normas reguladoras de la OIT Coercitividad que se ha reputado relativamente innecesaria en la medida en que el Tripartismo y los mecanismos de control inherentes tienen ciertos recorridos posibles en el control del cumplimiento de las normas.

No obstante lo anterior, sí es cierto que la OIT ha comenzado a desplegar nuevas formas de influencia, especialmente a partir de la Declaración relativa a los Principios y Derechos Fundamentales en el Trabajo y su Seguimiento, adop- 
tada por la Conferencia Internacional del Trabajo en su octogésima sexta reunión, Ginebra, 18 de junio de 1998 (Anexo revisado, 15 de junio de 2010).

A partir de esa fecha, desde la Organización Internacional del Trabajo se ha encontrado el modo de juridificar ciertos contenidos esenciales, que no son sino la rematerialización de contenidos ya presentes en el haber jurídico internacional (convenios OIT y derechos contenidos en el articulado de la Declaración Universal de Derechos Humanos). Y en este sentido, al margen de la aprobación de nuevos convenios sectoriales de gran relevancia (trabajadores del mar, servicio doméstico, son paradigmáticos) deviene singularmente trascendente la versión revisada en marzo 2017 de la Declaración tripartita de principios sobre las empresas multinacionales y la política social 5 .

Pues bien, de un modo particular, pero genéricamente centrado en el Trabajo y su Futuro, y próxima la fecha de su Centenario, la OIT ha impulsado a nivel mundial (a partir de la Memoria del Director General, presentada en la Conferencia Internacional del Trabajo, $104 .^{a}$ reunión, 2015) un amplio debate sobre el Futuro del Trabajo como respuesta a las dificultades que se plantean para abordar todas las repercusiones derivadas de un proceso en continua transformación en el mundo del trabajo (OIT, 2015).

Se constata que el mundo del trabajo hoy se caracteriza por varias circunstancias, a saber: una nueva relación entre empleo-pobreza y protección social, un fenómeno multiforme de internacionalización de la producción, y un retroceso en la calidad del trabajo (trabajo informal, vulneración de derechos humanos, explotación infantil y discriminaciones por razón de género). De una manera explícita, sin embargo, no se mencionan expresamente los nuevos cambios tecnológicos de la Industria 4.0; si bien están implícitas algunas intuiciones en la proyección reflexiva que se plantea.

En ese escenario la Memoria que presentara Guy Ryder en 2015 proponía cuatro conversaciones para ordenar el debate: 1) Trabajo y sociedad; 2) Trabajo decente para todos; 3) La organización del trabajo y de la producción; y 4) La gobernanza del trabajo. Conversaciones que provocan múltiples sendas discursivas acerca de los retos que el siglo xxi planteará para el Trabajo, y por ende para la OIT como organismo especializado de Naciones Unidas. Pero en paralelo, también en la OIT discurren otras seis iniciativas, como son: la Iniciativa para poner Fin a la Pobreza, la Iniciativa Verde, la Iniciativa relativa a las Mujeres en el Trabajo, la Iniciativa relativa a las Normas, la Iniciativa relativa a las Empresas y la Iniciativa sobre la Gobernanza.

5 Declaración tripartita de principios sobre las empresas multinacionales y la política social,

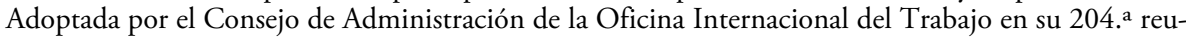

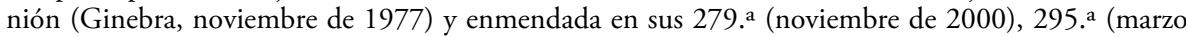
de 2006) y 329.a (marzo de 2017) reuniones, (http://www.ilo.org/wcmsp5/groups/public/---ed_ emp/---emp_ent/documents/publication/wcms_124924.pdf). 
De lo anterior se pueden atisbar cuáles son los ámbitos de las indefiniciones y vicisitudes actuales, en los que se está reclamando un proceso discursivoreflexivo nuevo e intensificado. Que se remite a los Estados, en su conformación tripartita (gobiernos-representantes empresariales y representantes sindicales) pero que ha de ser observada y participada por las Universidades; y, consecuentemente, por estudiantes, e investigadores-docentes.

\subsection{La Agenda ONU 2030 y los Objetivos del Desarrollo Sostenible como Axiomas duraderos de gestión necesaria del cambio}

Coetáneamente, desde la Organización de Naciones Unidas, con menor incidencia (puesto que su Organismo especializado es la OIT) también se ha empezado a prestar atención a cuestiones ligadas al Trabajo, especialmente en tanto es objeto y sujeto de los fenómenos de globalización económica, y en la medida en que los derechos laborales guardan una relación estrecha con los Derechos Humanos.

Estos, por su vis expansiva, se erigen en contenidos obligatorios no solo de los estados, sino también de las Empresas.

De esta forma, resultan relevantes son iniciativas. De una parte, el Informe del Representante Especial (John Ruggie) del Secretario General para la cuestión de los derechos humanos y las empresas transnacionales y otras empresas presentado en la Asamblea General de Naciones Unidad (2011) ${ }^{6}$. De otra parte, los Diez Principios Rectores del Pacto Mundial de Naciones Unidas (UN Global Compact ${ }^{7}$ ); de los cuales cuatro (principio 3-6 son principios específicamente laborales) si bien los demás inespecíficamente pueden tener incidencia laboral en la actividad de las Empresas.

En paralelo a lo anterior, y siempre de un modo lato, también se han de resaltar otros instrumentos internacionales con incidencia tangencial o directa sobre la reflexión del Futuro del Trabajo.

De un modo más concreto, dentro del primer objetivo de los Ocho Objetivos de Desarrollo del Milenio que conformaban la agenda 2015 (ODM 2015) de Naciones Unidas (del año 2000) el empleo y el trabajo decente ocupaba un

${ }^{6}$ Asamblea de Naciones Unidas, Informe del Representante Especial del Secretario General para la cuestión de los derechos humanos y las empresas transnacionales y otras empresas, John Ruggie. Principios Rectores sobre las empresas y los derechos humanos: puesta en práctica del marco de las Naciones Unidas para "proteger, respetar y remediar, 2011, A/HRC/17/31),

7 Cuyo objetivo es el de movilizar a un movimiento global de empresas sostenibles; de forma que apoya a las empresas para hacer negocios de manera responsable al alinear sus estrategias y operaciones con diez principios en materia de derechos humanos, trabajo, medio ambiente y lucha contra la corrupción; así como, desde 2015 también les ayuda a tomar acciones estratégicas para avanzar en los objetivos generales de la sociedad, tales como los Objetivos de Desarrollo Sostenible de la ONU, con énfasis en la colaboración y la innovación (https://www.unglobalcompact.org/what-is-gc) 
lugar primordial como instrumento de la erradicación del hambre y de la pobreza, especialmente referida al colectivo de mujeres; de un modo más potente desde el momento en que el Tercer ODM se dedicaba a la promoción de la igualdad por razón de género y el empoderamiento de la mujer. Y, en paralelo, la idea-fuerza de la Sostenibilidad también se encontraba presente en los trazados axiológicos internacionales, si bien circunscrita con cierta relación de esencialidad a la conservación del Medio Ambiente (7. ${ }^{\circ}$ ODM, 2015).

A partir de esos presupuestos, para 2030, la Asamblea General de Naciones Unida ha aprobado, bajo el titulo Transformar nuestro mundo: la Agenda 2030 para el Desarrollo Sostenible un proyecto de acción, anclado en 17 Objetivos de Desarrollo Sostenible (en adelante ODS, 2030).

Entre los Objetivos del desarrollo sostenible se encuentra uno directamente relacionado con el Trabajo (Objetivo 8 Trabajo decente y crecimiento económico); algunos con conexión tangencial pero no por ello menos relevantes ${ }^{8}$, alguno fuertemente transversales ${ }^{9}$, ciertos que se centran en ciertos sectores en los que el elemento jurídico-laboral tendría una vis expansiva muy importante ${ }^{10}$; y, por último, otros multivectoriales e instrumentales. ${ }^{11}$

Esa Agenda fue adoptada por unanimidad en la Asamblea General, y se sustenta en ciertos ejes conceptuales potentes: a saber: 1) A pesar de las formulaciones genéricas y aparentemente dispersas, los ODS y sus 163 Metas plantean compromisos efectivos a los Estados, 2) ODS y Metas se van a monitorizar y están sujetos a verificaciones cuantitativas regulares en términos de cumplimiento $^{12}$; 3) en la medida que los ODM ya pusieron de manifiesto la ineficacia de una visión clásico-estatista del cumplimiento internacional del Derecho Internacional Clásico ${ }^{13}$ y del Soft Law, desde la Agenda 2030 se hace una llamada a los Gobiernos/ Estados, pero también a la Sociedad Civil en general (Empresas, Tercer Sector...) y a la Comunidad Científica y Académica en particular.

8 ODS 1 Fin de la pobreza; ODS 3 Salud y Bienestar ; ODS 4 Educación de calidad; ODS 10 Reducción de las desigualdades; ODS 12 Producción y consumos responsables.

9 Como es el ODS 5 Lograr la igualdad de género y empoderar a todas las mujeres y las niñas,

10 ODS 6 Agua limpia y saneamiento; ODS 7 Energía asequible y no contaminante; ODS 9, Industria, innovación e infraestructura, ODS 11 Ciudades y comunidades sostenibles, ODS 13 Acción por el clima, ODS 14 Vida submarina, ODS 15 Vida de ecosistemas terrestres.

${ }^{11}$ Como los ODS 16 Paz, Justicia e Instituciones sólidas y ODS 17 Alianzas para lograr objetivos.

12 Ya se han formalizado en el Consejo económico y social de Naciones Unidas, a finales de 2016, los primeros indicadores. Data and indicators for the 2030 Agenda for Sustainable Development: Report of the Inter-agency and Expert Group on Sustainable Development Goal Indicators. (E/CN.3/2017/2*).

${ }^{13}$ En lo que se refiere, por ejemplo, monográficamente a los convenios y recomendaciones OIT, es muy descriptivo el elenco de ratificaciones por convenio y país. Que pone de manifiesto un cierto desdén por la normativa internacional (vid al respecto, http://www.ilo.org/dyn/normlex/ es/f?p=NORMLEXPUB:10011:0::NO::P10011_DISPLAY_BY,P10011_CONVENTION_TYPE_ CODE:1,F). 
De ahí que desde la Universidad (Sachs, (2015)), desde los planos docente y de investigación, quepa contribuir a esos objetivos mundiales, en un primer momento de una forma modesta, y quizás más adelante, de un modo más ambicioso.

\section{El papel de las Universidades como catalizadores de la investigación y de la formación en Objetivos de Desarrollo Sostenible en y para el Trabajo Decente}

La Implicación de la Universidad puede ser polimorfa, si bien está presente en el germen mismo del Desarrollo Sostenible.

Es en las universidades (centros Politécnicos de d todo el Mundo, con MIT a la cabeza, por señalar algún ejemplo) donde, paradójicamente, se ha gestado el desarrollo mismo de la Robotización y la Computerización y sus aplicaciones industriales. Y ha de ser en ellas donde, en tanto en cuanto son centros de investigación, pensamiento y desarrollo de conocimiento, se haya de llevar a cabo una actividad de reflexión sobre el Futuro del Trabajo.

Y aún más allá, a lo que aquí interesa, desde las Universidades, cabe avanzar en la investigación y clarificación de los ODS 2030 en las distintas ramas epistemológicas, por separado (Derecho, Economía, Ingeniería, Sociología etc.), pero también a través de redes de trabajo inter-multidisciplinares.

En momentos de transformación, como los que se están produciendo en un contexto de Revolución Industrial 4.0, la Universidad también es centro de difusión del conocimiento y de formación de profesionales. No solo en la faceta intrínsecamente sustantiva y adjetiva del Derecho Social, en lo que aquí interesa, sino también en la faceta ético-axiológica de la maestría jurídica. Y el Trabajo Decente y el Desarrollo Sostenible encajan perfectamente en esa faceta.

En efecto, si se observa, subyace al Desarrollo Sostenible una idea que supera la mera filantropía para construir una obligación moral en torno a la cohonestación del Desarrollo/ Crecimiento Económico con la Sostenibilidad entendida de un modo que supera la clásica de la sostenibilidad medioambiental porque incorpore una visión omnipotente de Equidad y Justicia social, que, por ejemplo, se materializa en la idea del Trabajo Decente/Digno. O dicho de otro modo, en la medida en que los ODS 2030 y sus metas son realidades jurídicas indeterminadas y difusas, desde la Universidad cabe corporeizarlas y reconducirlas a los esquemas jurídicos existentes.

Los ODS no crean necesariamente instituciones jurídico-económicas nuevas, sólo ofrecen un nuevo formato de la idea del Trabajo - Sostenible porque es Decente- que permite reformular derechos y obligaciones socio-laborales, 
de Derecho Laboral privado, pero de Derecho de Protección Social público. Pero también cabe inferir nuevos principios meta-jurídicos, capaces de servir de criterios interpretativos y aplicativos para la conformación de un Derecho Social crítico. Por poner algunos ejemplos:

a) La Sostenibilidad como no-afectación-omnímoda, puede aumentar el potencial del principio de la igualdad y la prohibición de discriminación de mujeres trabajadoras, de trabajadores jóvenes y maduros, de trabajadores migrantes; pero también se expande como axioma antidiscriminatorio en la condición de ciudadano de un eventual Estado de Bienestar.

b) La Sostenibilidad como no-abuso-de-poder imprime nueva fuerza a las demandas de lucha contra la pobreza en el trabajo, pero también en el uso/explotación de recursos naturales materiales diversos. La Sostenibilidad como Contrapartida para el progreso daría forma a las iniciativas de formación inclusiva o recualificadora de calidad, o al diseńo de políticas públicas y privadas de protección de la salud, reparadora o preventiva, en el trabajo/fuera del trabajo/ para el trabajo; femenina/infantil/de personas mayores...

c) La Sostenibilidad como fin-colectivo-último permitiría organizar las relaciones colectivas bajo nuevas formas. Cabría revisar la recuperación de zonas y ámbitos materiales negociales para los interlocutores sociales representativos (antiguos y/o nuevos), cabría establecer nuevas alianzas entre interlocutores sociales y poderes públicos, dentro de los esquemas ya existentes (pero bastante huecos), o proceder a la revisión de las formas arcaicas de responsabilidad social corporativa para reformular una Nueva Responsabilidad Social Empresarial.

La tarea de estudio y difusión de los ODS, y de formación sustantiva y ética a partir de ellos puede ser objeto de la labor investigadora individual y colectiva de los docentes, pero también puede ser objeto del aprendizaje consciente, mancomunado, colaborativo y sumativo de los estudiantes en el aula y fuera de ella, coadyuvados por aquéllos docentes investigadores.

En síntesis, en un contexto de cambio de paradigma del trabajo, los ODS son susceptibles de ser aproximados, en términos pedagógicos en los estudios universitarios, desde las perspectivas cognitiva (son contenidos), enactiva (es preciso actuarlos para comprobar su eficacia) y volitiva (son elementos externos de una nueva forma de motivación intrínseca). $Y$ esas tres vertientes encuentran un campo natural perfectamente claro, cual es el de los estudios de Relaciones Laborales. 


\section{Los estudios de Relaciones Laborales y los ODS}

Es cierto que los ODS son continentes de muchas materias extrajurídicas, y aun siendo jurídicas, de materias extra-laborales. Sin embargo, sí es verdad que, paralela y paradójicamente, el Trabajo y los aspectos ligados a él, a partir de una óptica laboro-céntrica son transversales a los 17 ODS y están presenten en una multiplicidad de las 163 Metas contenidas en la Agenda 2030.

\subsection{Los estudiantes como Agentes}

El Trabajo es el mimbre de las sociedades, de las empresas, de los Estados. Los ciudadanos lo son por pertenencia a una institución geopolítica, hoy nacional o con raigambre geográfica pero supranacional.

Y los Profesionales, los del Derecho y aquéllos con otras líneas de actividad de Gestión de Recursos Humanos, siempre han de estar ligados al Trabajo. Porque sea su obra personal, o porque gestionan obras ajenas (de las distintas maneras posibles, al ordenar, vender, transformar, conservar etc.). En esa visión Laboro-céntrica, desde la Universidad cabe inseminar capacidades de observación, y de construcción de realidades ligadas al Trabajo, ahora desde las líneas del Desarrollo sostenible.

De ahí que, quizás, entre los Grados más permeables a la contribución al Debate del Futuro del Trabajo, ensamblando los ODS como acelerantes, se encuentra el Grado en Relaciones Laborales y Empleo / y Recursos Humanos (en sus distintas terminologías universitarias). Porque los actuales estudiantes han de ser eventuales futuros profesionales del Trabajo y del Empleo ${ }^{14}$. $Y$ eso les demandaría una nueva óptica sensible a los desafíos jurídico-laborales y de la protección social, y un nuevo conocimiento crítico del Derecho Positivo vigente a partir de los ODS y de los Retos el Futuro del Trabajo.

En efecto, en el imaginario social general, hay hoy ciertos temas de actualidad que son aún un desafío en la práctica, pero también en el debate doctrinal, y que tienen un espacio natural universitario de composición teórico-práctica solvente en estos Grados de Relaciones Laborales. Temas como puedan ser los que se refieren a distintos aspectos del Estado del Bienestar genéricamente considerado (Renta Social Básica y trabajadores pobres, reformas de los sistemas de pensiones de jubilación y viudedad, protección por desem-

${ }^{14}$ En la Universidad Carlos III el grado se caracteriza por ser fuertemente multidisciplinar que proporciona herramientas conceptuales, técnicas y prácticas provenientes de una amplia variedad de materias, que habilitarán a los graduados a intervenir profesionalmente en importantes campos de actividad en todo tipo de empresas (dirección de recursos humanos, consultoría laboral, etc.), organizaciones no lucrativas (integración mediante el empleo, sindicatos, etc.) y sector público (oposiciones como la de Inspección de Trabajo o Subinspección de Empleo y Seguridad Social). 
pleo, protección de la dependencia, promoción de la atención a la infancia...); o como pudieran ser los que se refieren a aspectos más propios del Derecho Laboral Individual (control del tiempo de trabajo, regulación de salarios mínimos y /o dignos, eliminación de brechas de todo tipo por género y edad, integración de las diversidades en las empresas, la formación continua para el empleo, la protección contra la temporalidad en la contratación, el respeto de la intimidad, el afloramiento del trabajo Informal, prevención de riesgos laborales y promoción de la salud y el bienestar en la empresa, etc.); o del Derecho Laboral Colectivo (incidencia de las nuevas tecnologías en el ejercicio de los derechos de representación (legitimación, negociación colectiva, medidas de conflicto colectivo).

A la comprensión de esos desafíos se puede contribuir desde la docencia en el Grado de Relaciones Laborales y Empleo.

\subsection{Los ODS como marcos éticos del aprendizaje de los estudiantes}

Además se hace preciso desencadenar en los estudiantes de este grado un pensamiento crítico, en torno a la Realidad del Trabajo y a su Regulación Jurídica, que resulta cualitativa y cuantitativamente marginal. $Y$ es necesario porque el conocimiento crítico del Derecho Social les permitiría desempeñar su actividad profesional futura de un modo sosteniblemente responsable, es decir, sobre la óptica de la evaluación cuasi-automática de las implicaciones que la aplicación, interpretación, revisión de sus competencias y conocimientos profesionales tienen sobre el —clásicamente llamado- Capital humano presente, pero también futuro (trabajadores implicados en régimen de contratación directa o trabajadores presentes por efecto de la composición de cadenas de subcontratación, o por cesión legal, cadenas de contratación en fraude de ley con rotación subjetiva, por poner algunos ejemplos).

El diseño de condiciones de trabajo decentes y de derechos sociolaborales fortalecidos, la adecuada gestión de la diversidad, el respeto por la interiorización del Trabajo sin género, la pretensión de la justicia social como conditio sine qua non de progreso de cada una de las Naciones y del conjunto de $\mathrm{Na}$ ciones (y por tanto del Estado español, de las Comunidades Autónomas o las Corporaciones Locales), la eliminación de barreras artificiales incardinadas en las cadenas de valor, la promoción de la salud integral (física y mental) personalizada según actividad, género y edad, o la formación continua, son ejemplos de buenos efectos que podrían emanar de la cualificación universitaria de profesionales del /con el Derecho Social anclado en la Sostenibilidad entendida en ese sentido omnicompresivo que la haría efectiva en el medio-largo plazo. 


\subsection{Los ODS como herramientas de Innovación Docente}

\subsubsection{Nuevos contenidos: la afectación de los programas}

En este contexto se hace, entonces, necesario pensar en la revisión en mayor o menor profundidad de los programas de las distintas asignaturas del Grado y, en todo caso, en la necesidad de que los docentes actualicen, primero, sus conocimientos a través de investigaciones sustantivas concretas. Pero, segundo, actualicen sus competencias digitales y de aprehensión de los retos de la digitalización. Porque los estudiantes ya son profesionales potenciales de la nueva revolución industrial. Y pueden ser agentes de cambio, y de consolidación de derechos.

En la medida en que los calendarios del Espacio Europeo de Educación Superior en las distintas Universidades indican de éste se encuentra aún en fase de primera conformación y primeras evaluaciones por las Agencias autonómicas especializadas competentes, no sería deseable alterar significativamente los planes de estudio de los distintos grados y postgrados, ni ofertar aun nuevos títulos cuyo contenido exclusivo pudieran ser los ODS; algo que sí sería el objeto de una contribución universitaria ambiciosa. Pero sí es factible, aunque quizás menos impactante, la imbricación de los ODS en todos los Grados. Y por ello también en el de Relaciones Laborales y Empleo.

Así, desde la prudencia, sí cabe llevar a cabo algunas modificaciones en la enseñanza del Derecho Social, en orden a contribuir a optimizar los efectos de la Agenda 2030. En dos líneas, esas que ya se han avanzado más arriba, a saber: una más objetiva, ligadas a las conexiones jurídico-técnicas con las instituciones actualmente estudiadas en las distintas asignaturas de los planes de estudio. Y una más subjetiva, ligada a los aspectos ético-filosóficos emanantes del Derecho social considerado como un producto histórico social.

Desde una perspectiva objetiva, cabría, sin necesidad de modificar esencialmente los programas/fichas de las asignaturas, infiltrar conceptos de los ODS en los contenidos. Del mismo modo que se han hecho permeables los textos legales a actividades docentes que traspasan las labores meramente exegéticas y de aplicación mecánica-forense, y ahora las instituciones jurídicas se contextualizan en planos jurídicos, pero también socio-políticos y socio-económicos, cabría trasladar los contenidos de los Objetivos y metas de Desarrollo sostenible a las instituciones jurídicas existentes. Para valorar sus niveles de coincidencia, distancia, afinidad; en suma para valorar el recorrido que la regulación laboral y de protección social preexistentes presentan hacia la consecución de los desiderátums fijados por Naciones Unidas.

Desde un punto de vista subjetivo, desde las aulas universitarias cabe formar una nueva conciencia de la conformación actual del Mundo del Trabajo y de las distintas Alianzas que podrían conformarlo de un modo diferente, más equita- 
tivo, a partir de la consideración de los mandatos de los distintos ODS, 2030, pero erguidos sobre el pilar esencial del Trabajo Decente y Sostenible.

Cabe, una vez más, señalar algún ejemplo, y sirvan los siguientes. De un lado, a realidad de los trabajadores pobres ya está verificada y cuantificada desde hace algún tiempo (Blázquez, 2015 y Calvo, 2017), así como algunas de sus causas, casi todas ligadas a la última crisis económico-financiera, pero con raíces estructurales en el caso español (retrocesos de la negociación, bajos salarios, alta rotación laboral, déficits formativos, alta tasa de contratación temporal, abusos en la gestión empresarial del tiempo de trabajo, desinversión en políticas pública de protección social y retroceso en derechos prestacionales...). Los estudiantes del Grado en Relaciones Laborales han de adquirir conocimientos jurídicos sobre contratación, gestión de derechos salariales, regulación del tiempo de trabajo, régimen sancionador en casos de incumplimiento de normativa legal o convencional etc. En ese proceso, cabe infiltrar direccionamientos hacia diagnósticos de situación (como causa del status quo que estudian), y hacia metas de eventuales reformas legislativas (como efectos deseables en cumplimiento de ODS). En otras palabras habría un proceso reflexivo paralelo al del aprendizaje del Derecho Positivo vigente, en el que activarían los principios subyacentes que conducen la idea del Trabajo Decente, que sirva para huir de las situaciones de pobreza, porque hay salarios y cotizaciones dignas y, por reflejo, cotizaciones suficientes para estándares de protección alejados también del umbral de pobreza. En definitiva porque más allá de la compliance (que ya es deseable en este ámbito) se mejora el estatuto laboral de los trabajadores.

De otro lado, la igualdad de género es un ODS también transversal, y concomitante con un mandato constitucional español. De ahí que en la mayoría de las asignaturas, jurídicas y no estrictamente jurídica, queda adoptar una perspectiva de género en lo que se refiere a la intersección Trabajo (de hombres y mujeres)- Empresa, en orden a dar el máximo cumplimiento potencial de las normas existentes o, en su caso, minimizar el impacto de su aplicación en el incremento de las brechas distintas presentes (Sáez, 2016: 93 y ss. y Blázquez, 2017).

El Trabajo ha devenido una realidad múltiple (asalariado, por cuenta propia, asociativo, colaborativo...), susceptible de desplegarse como actividad más allá de la empresa tradicional, ubicada geográficamente en un único lugar; susceptible de organizarse de un modo tan flexible que se diluyen las coordenadas temporales clásicas. Pero, además, el Trabajo se concreta en actividades sujetas a un dinamismo desconocido, como consecuencia de las nuevas tecnologías amplo sensu y de sus potencialidades multivectoriales (nuevas tecnologías como objeto del Trabajo, como instrumento del Trabajo, como gestor del Trabajo...). Y todo eso ya se ha ido integrando, como contenido, en los programas universitarios. Sería entonces el momento de hacer lo propio con los contenidos de los 
ODS, 2030, para que los estudiantes puedan visualizar y comprender nuevas zonas de eventual avance futuro de las instituciones presentes.

Así, en la Universidad Carlos III de Madrid, ya hay algunos embriones en esa línea. Por un lado, en el curso 2016-17, en el marco de una asignatura de Humanidades, titulada Género y protección social tras la crisis ${ }^{15}$, ya se ha introducido, como instrumento de análisis transversal del diagnóstico, de los contornos de la situación actual de la protección de la mujer (trabajadora o no) desde las distintas políticas públicas y de los comportamientos empresariales, el ODS 5. Y los estudiantes han auditado ciertas materias (salario, formación, conciliación, pensiones) desde las ópticas normativas internas y autonómicas, pero también con las metas incluidas en este Objetivo.

Por otro lado, ya ha sido aprobado un curso MOOC titulado Strategies for the implementation of sustainable Work at companies, para el próximo curso 2017-2018, en el que se pretenden afrontar los modos en que los distintos ODS, 2030 pueden ser implementados en el marco de empresas socio-laboralmente responsables. Y habría de actuar como actividad docente piloto en este sentido.

De la misma manera, en las fases de revisión de los distintos grados de la Universidad Carlos III de Madrid, y también entonces del Grado en Relaciones Laborales y Empleo, se está produciendo un debate en torno a la necesidad de revisar títulos y contenidos de ciertas asignaturas, en orden a incluir en las fichas oficiales conceptos y contenidos ligados los objetivos internacionales (ODS. 2030 y nuevos retos del Futuro del Trabajo), tales como la Gobernanza Social como nuevo marco del Dialogo social, la Responsabilidad Social Corporativa como contenido de la actividad profesional/empresarial más allá del mero beneficio económico; la revisión de los conceptos de Necesidad ligados a la Pobreza dentro de los esquemas de protección social publica y complementaria, como primeros paradigmas.

\subsubsection{Fundamentos Profesionales éticos}

Junto a esa vertiente más objetiva, de los nuevos contenidos docentes en distintos grados, también cabria definir un objetivo ambicioso en términos subjetivos. En orden a conformar profesionales sensibles a los ODS, 2030, que actúen como agentes reales de su implementación, siquiera a pequeńa escala.

En suma, se trataría de que la enseñanza universitaria, con sustrato en actividades de investigación ligadas al desarrollo (socio-económico-político-cultural) sostenible fuera un instrumento germinal de futuros profesionales comprometi-

15 Inicialmente prevista para $2 .^{\circ}$ cuatrimestre, para estudiantes de $1 .^{\circ}$ Economía, quedo abierta a estudiantes del Grado en Relaciones Laborales y Empleo, de $1 .^{\circ}, 2 .^{\circ}$ y tercer Curso. 
dos con una visión Global del Trabajo y, en suma, del futuro de derechos sociales subjetivos, efectivos, polivalentes, transnacionales. Porque habrá que gestionar una Transición /Revolución Digital con efectos en el empleo, y los ODS pueden ser guías para hacerlo en el medio/largo plazo.

\subsubsection{La Motivación de los estudiantes}

La Agenda 2030 de Naciones Unidas contiene 17 Objetivos de Desarrollo Sostenible (ODS) que, además de ser líneas directrices para los Estados, demandan la intervención de la sociedad civil en su consecución. Y las Universidades se encuentran allí llamadas a colaborar en la difusión e implementación de los ODS y sus 163 metas. Y los - hoy- estudiantes son los ciudadanos, profesionales, empresarios, empleados públicos del futuro, de ese horizonte 2030.

Como se ha venido reiterando, los ODS son marcos ambiciosos, pero difusos, lo que supone tanto un riesgo en orden a su efectividad, cuanto una ventaja atractiva de cara a su potencial desarrollo. No están sujetos a definiciones preconceptualizadas o preconcebidas. Solo tienen un elemento relativamente rígido en la medida en que, a diferencia de los ODM, en la Agenda 2030 se ha incluido una garantía relativa de eficacia, concretada en la definición de indicadores (referentes cuantitativos) de impacto/medida del grado de consecución de objetivos y metas. De un modo inespecífico, habría entonces una cierta definición de los resultados comprometidos respecto de los Estados para con Naciones Unidas, pero no hay exigencias de los modos de conseguir alcanzar los índices previstos.

Esa ductilidad/versatilidad/potencialidad de los ODS resulta óptima como elemento innovador de motivación. Especialmente si se entiende que el aprendizaje más potente se produce a partir del sistema límbico (Maclean, 1990), y como ha ido quedando acreditado desde la neurociencia y la neuropsicología (sin que sea posible en este trabajo mayor detenimiento), la emoción contribuye a un mayor aprendizaje significativo (Mora 2013).

Esto es muy relevante porque, en un determinado contexto político, social y económico, los estudiantes universitarios, con excepciones siempre, no quedan ajenos a una relativamente frecuente apatía / descreimiento, tedio, desmotivación en suma, respecto de ciertas materias de estudio. De ahí que, como Experiencia Piloto, algunas asignaturas ligadas al Derecho Social podrían servir para testar si esos ODS sirven para provocar reflexión sobre el objeto de estudio, a la vez que dinamiza a los estudiantes y los hace participes de la creación de un nuevo foro de acción y pensamiento en torno a los ODS y su potencial social.

Los Trabajos Fin de Grado o las asignaturas ligadas a la igualdad de las mujeres (ODS 5) y al Trabajo amplo sensu (ODS 3,4,8,10) serían continentes óptimos para insertar un nuevo modo de motivación a los estudiantes: el de poder, 
desde el Grado en Relaciones Laborales, en el marco de los ODS, confrontarles la lege data, para proponer una nueva construcción. En efecto, con distintos formatos, en distintos foros posibles (en las distintas actividades universitarias), cabría que pudieran construir, desde la Universidad, como estudiantes y potenciales ciudadanos, algo real que proponer a la academia universitaria, a los poderes públicos (distintas administraciones), a los agentes políticos y a los interlocutores sociales. Como estudiantes podrían verter conocimiento lege ferenda a la sociedad. En la medida en que la Agenda 2030 necesita difundirse, pensarse, y transformar la realidad actual — la del Trabajo a lo que aquí concierne- cabe que los estudiantes se puedan sentir estimulados por la posibilidad de generar conocimiento/reflexiones disruptivas. Tanto más cuando las distintas reformas laborales han ido alejando al Derecho Social de ciertos ODS, en una deriva regresiva, cuasi expropiatoria de derechos sociolaborales.

Especialmente estimulante podría resultar la actividad clínica como método docente, porque ofrece posibilidades de activación, a través del desarrollo de actividades de auditoría, elaboración de informes, difusión de resultados ligados a los ODS.

De esta forma, de un modo sobrevenido quizás, los ODS puedan estar presentes en las tres dimensiones del aprendizaje, en tanto son contenido material, referente ético e instrumento de motivación. Profesores, profesionales, empresas $\mathrm{y}$ universidad han de hacer su papel en este proceso, se supone.

\section{Bibliografía}

Atkinson, Robert \& Wu, John (2017): «False Alarmism: Technological Disruption and the U.S. Labor Market, 1850-2015», ITIF @work series, mayo.

Barreto, Hugo (2017): «Tres determinismos tecnológicos sobre el futuro del trabajo», http://hugobarrettoghione.blogspot.com.es/2017/07/tres-determinismostecnologicos-sobre.html.

Blázquez Agudo, Eva María (2015): El trabajador pobre como centro de gravedad de la prevención de la exclusión. Albacete, Bomarzo.

Blázquez Agudo, Eva María (Coord.) (2017): Prestaciones de la Seguridad Social y género. Madrid: Organización Iberoamericana De Seguridad Social (OISS). 2017. E-archivo. http://www.oiss.org/IMG/pdf/PRESTACIONES_DE_LA_SEGURIDAD_ SOCIAL_Y_GENERO.pdf.

Calvo Gallego, Francisco Javier y Gómez Álvarez, María Rosario (Dirs.) (2017): Trabajadores pobres y pobreza en el trabajo. Murcia, Laborum.

FreY, Karl Benedikt y Osborn, Michael A. (2017): "The future of employment: How susceptible are jobs to computerization?», Technological Forecasting \& Social Change, 114, 254-280.

FreEman, Richard B. (2015): «Who owns the robots rules the world», IZA World of Labor, 5. 
Ford, Martin (2016): El Auge de los robots. Madrid, Paidos

KaPlan, Jerry (2016): Artificial Intelligence, Oxford, Oxford University Press.

LANTARón BARquín, David (2017): «Estándares laborales en la era de la globalización», Trabajo y Derecho, 4 (35-56).

López García, Pedro (2016): «La digitalización en el mundo del trabajo». Estudios, Fundación $1 .{ }^{\circ}$ Mayo, 9 , julio (1-16).

Maclean, Paul D. (1990): The triune brain in evolution: Role in paleocerebral functions. New York, Plenum Press.

McKinsey Global Institute (2017): A Future that Works: automation, employment and productivity (enero) (http://www.mckinsey.com/global-themes/digitaldisruption/harnessing-automation-for-a-future-that-works).

Mercader Uguina, Jesús R. (2017): El futuro del trabajo en la era de la digitalización y la robótica. Valencia, Tirant lo Blanch.

Ministerio de Industria, Energía y Turismo (2015): Industria Conectada 4.0. La transformación digital de la industria (http://www6.mityc.es/IndustriaConectada40/ informe-industria-conectada40.pdf).

Mora, Francisco (2013): Neuroeducación. Ed. Alianza Editorial.

Organización InTERnaCional del Trabajo (2015): La iniciativa del centenario relativa al futuro del trabajo, Ginebra, Oficina Internacional del Trabajo.

Roland Berger \& Siemens España (2016): España 4.0. El reto de la transformación digital de la economía (http://w5.siemens.com/spain/web/es/estudiodigitalizacion/ Documents/Estudio_Digitalizacion_Espana40_Siemens.pdf).

SACHS, Jeffrey (2015): The Age of sustainable Development, Columbia University Press.

SÁEz LARA, Carmen (Coord.) (2016): Igualdad de género: estrategias y propuestas. Murcia, Laborum.

Schwab, Klaus (2016): La cuarta revolución industrial. Madrid, Debate.

Todoli, Adrian (2017): El trabajo en la era de la económica colaborativa. Valencia, Tirant lo Blanch.

World Economic Forum (2016): The Future of Jobs. Employment, Skills and Workforce Strategy for the Fourth Industrial Revolution, enero(http://www3.weforum.org/docs/ WEF_FOJ_Executive_Summary_Jobs.pdf). 